\title{
Half-Step Continuous Block Method for the Solutions of Modeled Problems of Ordinary Differential Equations
}

\author{
A. A. James ${ }^{1}$, A. O. Adesanya ${ }^{2}$, J. Sunday ${ }^{3}$, D. G. Yakubu ${ }^{4}$ \\ ${ }^{1}$ Department of Mathematics, American University of Nigeria, Yola, Nigeria \\ ${ }^{2}$ Department of Mathematics, Modibbo University of Technology, Yola, Nigeria \\ ${ }^{3}$ Department of Mathematical Sciences, Adamawa State University, Mubi, Nigeria \\ ${ }^{4}$ Department of Mathematics, Tafawa Balewa Federal University of Bauchi, Bauch State, Nigeria \\ Email: adewale.james@aun.edu.ng
}

Received July 27, 2013; revised August 29, 2013; accepted September 18, 2013

Copyright (C) 2013 A. A. James et al. This is an open access article distributed under the Creative Commons Attribution License, which permits unrestricted use, distribution, and reproduction in any medium, provided the original work is properly cited.

\begin{abstract}
In this paper, we developed a new continuous block method using the approach of collocation of the differential system and interpolation of the power series approximate solution. A constant step length within a half step interval of integration was adopted. We evaluated at grid and off grid points to get a continuous linear multistep method. The continuous linear multistep method is solved for the independent solution to yield a continuous block method which is evaluated at selected points to yield a discrete block method. The basic properties of the block method were investigated and found to be consistent and zero stable hence convergent. The new method was tested on real life problems namely: SIR model, Growth model and Mixture Model. The results were found to compete favorably with the existing methods in terms of accuracy and error bound.
\end{abstract}

Keywords: Approximate Solution; Interpolation; Collocation; Half Step; Converges; Block Method

\section{Introduction}

We consider the numerical solution of first order initial value problems of the form:

$$
y^{\prime}=f(x, y), y\left(x_{0}\right)=y_{0}
$$

where $f$ is continuous and satisfies Lipchitz's condition that guarantees the uniqueness and existence of a solution.

Problem in the form (1) has wide application in physical science, engineering, economics, etc. Very often, these problems do not have an analytical solution, and this has necessitated the deviation of numerical schemes to approximate their solutions.

In the past, scholars have developed a continuous linear multistep in solving (1). These authors proposed methods with different basis functions and among them were [1-6] to mention a few.

These authors proposed methods ranging from predictor corrector method to discrete block method.

Scholars later proposed block method. This block method has the properties of Runge-kutta method for being self-starting and does not require development of separate predictors or starting values. Among these au- thors are [7-12]. Block method was found to be cost effective and gave better approximation.

This paper is divided into sections as follows: Section 1 is the introduction and background of the study; Section 2 contains the discussion about the methodology involved in deriving the continuous multistep method and the continuous block method. Section 3 considers the analysis of the block method in terms of the order, zero stability and the region of absolute stability. Section 4 focuses on the application of the new method on some numeric examples and Section 5 is on the discussion of result. We tested our method on first order ordinary differential equations and compared our result with existing methods.

\section{Methodology}

Consider power series approximate solution in the form

$$
y(x)=\sum_{j=0}^{s+r-1} a_{j} x^{j}
$$

where $S$ and $r$ are the number of interpolation and collocation points respectively. 
The first derivative of (2) gives

$$
y^{\prime}(x)=\sum_{j=0}^{s+r-1} j a_{j} x^{j-1}
$$

Substituting (3) into (2) gives

$$
f(x, y)=\sum_{j=0}^{s+r-1} j a_{j} x^{j-1}
$$

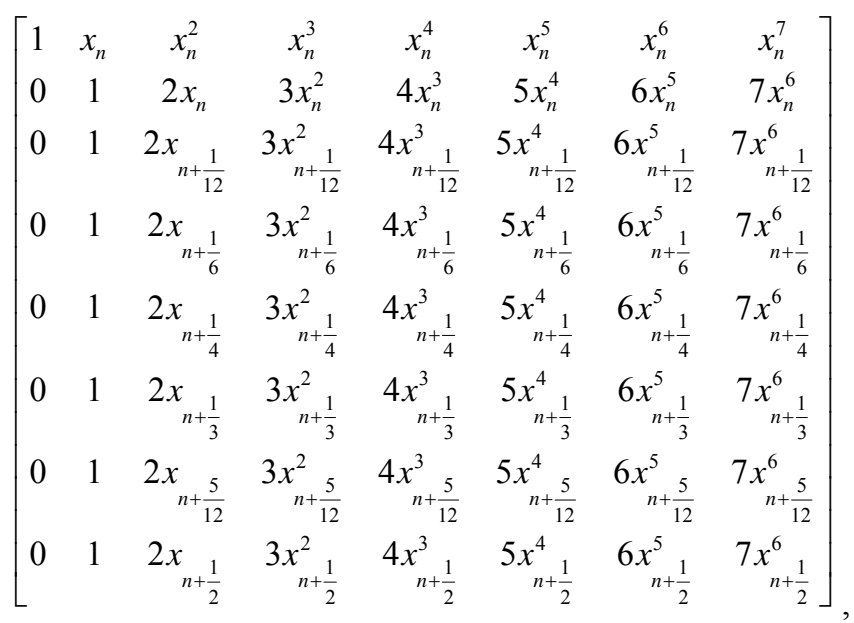$$
A=\left[\begin{array}{l}
a_{0} \\
a_{1} \\
a_{2} \\
a_{3} \\
a_{4} \\
a_{5} \\
a_{6} \\
a_{7}
\end{array}\right], U=\left[\begin{array}{c}
y_{n} \\
f_{n} \\
f_{n+\frac{1}{12}} \\
f_{n+\frac{1}{6}} \\
f_{n+\frac{1}{4}} \\
f_{n+\frac{1}{3}} \\
f_{n+\frac{5}{12}} \\
f_{n+\frac{1}{2}}
\end{array}\right]
$$
form

$$
A X=U
$$

where

Collocating (3) at $x_{n+s}, s=0\left(\frac{1}{12}\right) \frac{1}{2}$ and interpolating (2) at $x_{n}$ gives a system of non-linear equation in the

Solving (5) for the $a_{j}$ s and substituting back into (4) where $a_{0}=1$ and the coefficients of $f_{n+j}$ gives gives a continuous multistep method in the form

$$
\begin{aligned}
& y(x)=\alpha_{0} y_{n}+h \sum_{j=0}^{\frac{1}{2}} \beta_{j}(x) f_{n+j}, j=0\left(\frac{1}{12}\right) \frac{1}{2} \\
& \beta_{0}=\left(\frac{1}{120}\right)\left(124416 t^{7}-254016 t^{6}+211680 t^{5}-92610 t^{4}+22736 t^{3}-3087 t^{2}+210 t\right) \\
& \beta_{\frac{1}{12}}=\left(-\frac{1}{35}\right)\left(-124416 t^{7}-241920 t^{6}-187488 t^{5}+73080 t^{4}-14616 t^{3}+1260 t^{2}\right) \\
& \beta_{\frac{1}{6}}=\left(\frac{1}{35}\right)\left(311040 t^{7}-57456 t^{6}+414288 t^{5}-145215 t^{4}+24570 t^{3}-1575 t^{2}\right) \\
& \beta_{\frac{1}{4}}=\left(-\frac{1}{105}\right)\left(-1244160 t^{7}-2177280 t^{6}-1463616 t^{5}+468720 t^{4}-71120 t^{3}+4200 t^{2}\right) \\
& \beta_{\frac{1}{3}}=\left(\frac{1}{70}\right)\left(622080 t^{7}-1028160 t^{6}+647136 t^{5}-193410 t^{4}+27720 t^{3}-1575 t^{2}\right) \\
& \beta_{\frac{5}{12}}=\left(-\frac{1}{35}\right)\left(-124416 t^{7}+193536 t^{6}-114912 t^{5}+32760 t^{4}-4536 t^{3}+252 t^{2}\right) \\
& \beta_{\frac{1}{2}}=\left(\frac{1}{105}\right)\left(62208 t^{7}-90720 t^{6}+51408 t^{5}-14175 t^{4}+1918 t^{3}-105 t^{2}\right)
\end{aligned}
$$


where $t=\frac{x-x_{n}}{h}$. Solving (6) for the independent solution gives a continuous block method in the form

$$
y_{n+k}=\sum_{j=0}^{\mu-1} \frac{(j h)^{m}}{m !} y_{n}^{(m)}+h^{\mu} \sum_{j=0}^{s} \sigma_{j}(x) f_{n+j}
$$

where $\mu$ is the order of the differential equation $s$ is the collocation points. Hence the coefficient of $f_{n+j}$ in (7)

$$
\begin{aligned}
\sigma_{0}= & \left(\frac{1}{120}\right)\left(124416 t^{7}-254016 t^{6}+211680 t^{5}\right. \\
& \left.-92610 t^{4}+22736 t^{3}-3087 t^{2}+210 t\right) \\
\sigma_{\frac{1}{12}}= & \left(-\frac{1}{35}\right)\left(-124416 t^{7}-241920 t^{6}-187488 t^{5}\right. \\
& \left.+73080 t^{4}-14616 t^{3}+1260 t^{2}\right) \\
\sigma_{\frac{1}{6}}= & \left(\frac{1}{35}\right)\left(311040 t^{7}-57456 t^{6}+414288 t^{5}\right. \\
& \left.-145215 t^{4}+24570 t^{3}-1575 t^{2}\right) \\
\sigma_{\frac{1}{4}}= & \left(-\frac{1}{105}\right)\left(-1244160 t^{7}-2177280 t^{6}-1463616 t^{5}\right. \\
& \left.+468720 t^{4}-71120 t^{3}+4200 t^{2}\right) \\
\sigma_{\frac{1}{3}}= & \left(\frac{1}{70}\right)\left(622080 t^{7}-1028160 t^{6}+647136 t^{5}\right. \\
& \left.+193410 t^{4}+27720 t^{3}-1575 t^{2}\right) \\
& \left(-\frac{1}{35}\right)\left(-124416 t^{7}+193536 t^{6}-114912 t^{5}\right. \\
& \left.+3276 t^{4}-4536 t^{3}+252 t^{2}\right)
\end{aligned}
$$

$$
\begin{gathered}
\sigma_{\frac{1}{2}}=\left(\frac{1}{105}\right)\left(62208 t^{7}-90720 t^{6}+51408 t^{5}\right. \\
\left.-14175 t^{4}+1918 t^{3}-105 t^{2}\right)
\end{gathered}
$$

where $t=\frac{x-x_{n}}{h}$.

Evaluating (2.5) at $t=\frac{1}{12}\left(\frac{1}{12}\right) \frac{1}{2}$ gives a discrete block formula of the form

$$
Y_{m}=e y_{n}+h d f\left(y_{n}\right)+h d f\left(Y_{m}\right)
$$

where $e, d$, are $r \times r$ matrix

where

$$
\begin{gathered}
d \\
=\left[\begin{array}{llllll}
\frac{19087}{725760} & \frac{1139}{45360} & \frac{137}{5376} & \frac{143}{5670} & \frac{3715}{145152} & \frac{41}{1680}
\end{array}\right]^{\mathrm{T}} \\
Y_{m}=\left[\begin{array}{lll}
y_{n+\frac{1}{12}}, y_{n+\frac{1}{6}}, y_{n+\frac{1}{4}}, y_{n+\frac{1}{3}}, y_{n+\frac{5}{12}}, y_{n+\frac{1}{2}}
\end{array}\right]^{\mathrm{T}}
\end{gathered}
$$$$
e=\left[\begin{array}{llllll}
0 & 0 & 0 & 0 & 0 & 1 \\
0 & 0 & 0 & 0 & 0 & 1 \\
0 & 0 & 0 & 0 & 0 & 1 \\
0 & 0 & 0 & 0 & 0 & 1 \\
0 & 0 & 0 & 0 & 0 & 1 \\
0 & 0 & 0 & 0 & 0 & 1
\end{array}\right]
$$

$$
b=\left[\begin{array}{cccccc}
\frac{2713}{30240} & \frac{-15487}{241920} & \frac{293}{5670} & \frac{-6737}{241920} & \frac{263}{30240} & \frac{-863}{725760} \\
\frac{47}{378} & \frac{11}{15120} & \frac{83}{2835} & \frac{-269}{15120} & \frac{11}{1890} & \frac{-37}{45360} \\
\frac{27}{224} & \frac{387}{8960} & \frac{17}{210} & \frac{-243}{8960} & \frac{9}{1120} & \frac{-29}{26880} \\
\frac{116}{945} & \frac{32}{945} & \frac{376}{2835} & \frac{29}{1890} & \frac{4}{945} & \frac{-2}{2835} \\
\frac{725}{6048} & \frac{2125}{48384} & \frac{125}{1134} & \frac{3875}{48384} & \frac{235}{6048} & \frac{-275}{145152} \\
\frac{9}{70} & \frac{9}{560} & \frac{17}{105} & \frac{9}{560} & \frac{9}{70} & \frac{41}{1680}
\end{array}\right]
$$




\section{Analysis of the Basic Properties of Our New Method}

\subsection{Order of the Method}

Let the linear operator $L\{y(x): h\}$ associated with the block formular be defined as

$$
L\{y(x): h\}=A^{(0)} Y_{m}-e y_{n}-h^{\mu} d f\left(y_{n}\right)-h^{\mu} b F\left(Y_{m}\right)
$$

expanding in Taylor series and comparing the coefficient of $\mathrm{h}$ gives

$$
\begin{aligned}
L\{y(x) ; h\}= & c_{0} y(x)+c_{1} h y^{\prime(x)}+c_{1} h y^{\prime \prime(x)} \cdots c_{p} h^{p} y^{p}(x) \\
& +c_{p+1} h^{p+1} y^{p+1}(x)+c_{p+2} h^{p+2} y^{p+2}
\end{aligned}
$$

Definition:-The linear operator $L$ and the associated continuous linear multistep method (3.1) are said to be of order $\mathrm{p}$ if $c_{0}=c_{1}=c_{2}=\cdots=c_{p}=0$ and $c_{p+1} \neq 0$ is called the error constant and implies that the local truncation error is given by

$$
t_{n+k}=C_{p+2} h^{p+1} y^{p+1}\left(x_{n}\right)+0\left(h^{p+2}\right) \text {. }
$$

For our method;

$$
L\{y(x) ; h\}=\left[\begin{array}{c}
y_{n+\frac{1}{12}} \\
y_{n+\frac{1}{6}} \\
y_{n+\frac{1}{4}} \\
y_{n+\frac{1}{3}} \\
y_{n+\frac{5}{12}} \\
y_{n+\frac{1}{2}}
\end{array}\right]-\left[\begin{array}{cc}
1 & \frac{1}{12} h \\
1 & \frac{1}{6} h \\
1 & \frac{1}{4} h \\
1 & \frac{1}{3} h \\
1 & \frac{5}{12} h \\
1 & \frac{1}{2} h
\end{array}\right]\left[y_{n}\right]-\left[\begin{array}{c}
f_{n} \\
f_{n+\frac{1}{12}} \\
f_{n+\frac{1}{6}} \\
f_{n+\frac{1}{4}} \\
f_{n+\frac{1}{3}} \\
f_{n+\frac{5}{12}} \\
f_{n+\frac{1}{2}}
\end{array}\right]=0
$$

Expanding in Taylor series expansion gives

$$
\begin{aligned}
& \sum_{j=0}^{\infty} \frac{\left(\frac{1}{12}\right)^{j}}{j !} y_{n}^{j}-y_{n}-\frac{19087}{725760} h y_{n}^{\prime}-\sum_{j=0}^{\infty} \frac{h^{j+1}}{j !} y_{n}^{j+1}\left\{\frac{2713}{30240}\left(\frac{1}{12}\right)^{j}+\frac{15487}{241920}\left(\frac{1}{6}\right)^{j}\right. \\
& \left.-\frac{293}{5670}\left(\frac{1}{4}\right)^{j}+\frac{6737}{241920}\left(\frac{1}{3}\right)^{j}+\frac{263}{30240}\left(\frac{5}{6}\right)^{j}-\frac{863}{725760}\left(\frac{1}{2}\right)^{j}\right\} \\
& \sum_{j=0}^{\infty} \frac{\left(\frac{1}{6}\right)^{j}}{j !} y_{n}^{j}-y_{n}-\frac{1139}{45360} h y_{n}^{\prime}-\sum_{j=0}^{\infty} \frac{h^{j+1}}{j !} y_{n}^{j+1}\left\{\frac{47}{378}\left(\frac{1}{12}\right)^{j}+\frac{11}{15120}\left(\frac{1}{6}\right)^{j}\right. \\
& \left.+\frac{83}{2835}\left(\frac{1}{4}\right)^{j}-\frac{269}{15120}\left(\frac{1}{3}\right)^{j}-\frac{11}{1890}\left(\frac{5}{12}\right)^{j}-\frac{37}{45360}\left(\frac{1}{2}\right)^{j}\right\} \\
& \sum_{j=0}^{\infty} \frac{\left(\frac{1}{4}\right)^{j}}{j !} y_{n}^{j}-y_{n}-\frac{137}{5376} h y_{n}^{\prime}-\sum_{j=0}^{\infty} \frac{h^{j+1}}{j !} y_{n}^{j+1}\left\{\frac{27}{224}\left(\frac{1}{12}\right)^{j}+\frac{387}{8960}\left(\frac{1}{6}\right)^{j}\right. \\
& \left.+\frac{17}{210}\left(\frac{1}{4}\right)^{j}-\frac{243}{8960}\left(\frac{1}{3}\right)^{j}+\frac{9}{1120}\left(\frac{5}{12}\right)^{j}-\frac{29}{26880}\left(\frac{1}{2}\right)^{j}\right\} \\
& +\frac{\left(\frac{1}{3}\right)^{j}}{2835} y_{n}^{j}-y_{n}-\frac{143}{5670} h y_{n}^{\prime}-\sum_{j=0}^{\infty} \frac{h^{j+1}}{j !} y_{n}^{j+1}\left\{\frac{116}{945}\left(\frac{1}{12}\right)^{j}+\frac{32}{945}\left(\frac{1}{6}\right)^{j}\right. \\
& \left.\sum_{j=0}^{j} \frac{29}{1890}\left(\frac{1}{3}\right)^{j}+\frac{4}{945}\left(\frac{5}{12}\right)^{j}-\frac{2}{2835}\left(\frac{1}{2}\right)^{j}\right\}
\end{aligned}
$$




$$
\begin{aligned}
& \sum_{j=0}^{\infty} \frac{\left(\frac{5}{12}\right)^{j}}{j !} y_{n}^{j}-y_{n}-\frac{3715}{145152} h y_{n}^{\prime}-\sum_{j=0}^{\infty} \frac{h^{j+1}}{j !} y_{n}^{j+1}\left\{\frac{725}{6048}\left(\frac{1}{12}\right)^{j}+\frac{2125}{48384}\left(\frac{1}{6}\right)^{j}\right. \\
& \left.+\frac{125}{1134}\left(\frac{1}{4}\right)^{j}+\frac{3875}{48384}\left(\frac{1}{3}\right)^{j}+\frac{235}{6048}\left(\frac{5}{12}\right)^{j}-\frac{275}{145152}\left(\frac{1}{2}\right)^{j}\right\} \\
& \sum_{j=0}^{\infty} \frac{\left(\frac{1}{2}\right)^{j}}{j !} y_{n}^{j}-y_{n}-\frac{41}{1680} h y_{n}^{\prime}-\sum_{j=0}^{\infty} \frac{h^{j+1}}{j !} y_{n}^{j+1}\left\{\frac{9}{70}\left(\frac{1}{12}\right)^{j}+\frac{9}{560}\left(\frac{1}{6}\right)^{j}\right. \\
& \left.+\frac{17}{105}\left(\frac{1}{4}\right)^{j}+\frac{9}{560}\left(\frac{1}{3}\right)^{j}+\frac{9}{70}\left(\frac{5}{12}\right)^{j}-\frac{41}{1680}\left(\frac{1}{2}\right)^{j}\right\}
\end{aligned}
$$

Equating coefficients of the Taylor series expansion to zero yield

$$
\begin{aligned}
c_{0}=c_{1}=\cdots=c_{6}=0 \\
c_{7}=[3.78(-09) 2.53(-09) 3.49(-09) 1.85(-09) 1.68(-08) 5.56(-08)]
\end{aligned}
$$

Hence we arrived at a uniform order 6 for our method with error constants

\subsection{Zero Stability}

Definition: The block (8) is said to be zero stable, if the roots $\mathrm{Zs}, \quad s=1,2, \cdots, N$ of the characteristic polynomial $\rho(z)$ defined by $\rho(z) \operatorname{det}\left(z A^{(0)}-E\right)$ satisfies $\left|z_{s}\right| \leq 1$ and every root satisfying $\left|z_{s}\right| \leq 1$ have multiplicity not exceeding the order of the differential equation. Moreover as $h \rightarrow 0, \rho(z)=z^{r-\mu}(z-1)^{\mu}$ where $\mu$ is the order of the differential equation, $r$ is the order of the matrix $A^{(0)}$ and E.

For our method

$$
\rho(z)=\left[z\left[\begin{array}{llllll}
1 & 0 & 0 & 0 & 0 & 0 \\
0 & 1 & 0 & 0 & 0 & 0 \\
0 & 0 & 1 & 0 & 0 & 0 \\
0 & 0 & 0 & 1 & 0 & 0 \\
0 & 0 & 0 & 0 & 1 & 0 \\
0 & 0 & 0 & 0 & 0 & 1
\end{array}\right]-\left[\begin{array}{llllll}
0 & 0 & 0 & 0 & 0 & 1 \\
0 & 0 & 0 & 0 & 0 & 1 \\
0 & 0 & 0 & 0 & 0 & 1 \\
0 & 0 & 0 & 0 & 0 & 1 \\
0 & 0 & 0 & 0 & 0 & 1 \\
0 & 0 & 0 & 0 & 0 & 1
\end{array}\right]\right]=0
$$

$\rho(z)=z^{5}(z-1)$. Hence our method is zero stable.

\subsection{Region of Absolute Stability}

The block formulated as a general linear method where it is partition in the form

$$
\left[\begin{array}{c}
Y \\
Y_{n-i}
\end{array}\right]=\left[\begin{array}{ll}
A_{1} & B_{1} \\
A_{2} & B_{2}
\end{array}\left[\begin{array}{c}
h f(y) \\
y_{n}
\end{array}\right]\right]
$$

The elements of $A_{1}$ and $A_{2}$ are obtained from the coefficients of the collocation points, $B_{1}$ and $B_{2}$ are obtained from the interpolation points.

Applying the test equation $y^{\prime}=\lambda y$ leads to the re- currence equation

$$
y^{i+1}=M(Z) y^{i}, Z=\lambda h, i=1,2, \cdots, \mu-1
$$

The stability function is given by

$$
M(Z)=B_{2}+Z A_{2}\left(I-Z A_{1}\right)^{-B_{1}}
$$

and the stability polynomial of the method is given as

$$
\rho(\lambda, Z)=\operatorname{det}(\lambda I-M(Z))
$$

The region of absolute stability of the method is defined as $\rho(\lambda, Z)=1,|\lambda| \leq 1$.

For our method, writing the block in partition form gives 


$$
\begin{aligned}
& {\left[\begin{array}{ccccccccc}
y_{n} y_{n+\frac{1}{12}} y_{n+\frac{1}{6}} y_{n+\frac{1}{4}} y_{n+\frac{1}{3}} y_{n+\frac{5}{12}} y_{n+1}
\end{array}\right]^{\mathrm{T}}} \\
& {\left[\begin{array}{cccccccccc}
0 & 0 & 0 & 0 & 0 & 0 & 0 & \cdots & 0 & 1 \\
\frac{19087}{725760} & \frac{2713}{30240} & \frac{-15487}{241920} & \frac{293}{5670} & \frac{-6737}{241920} & \frac{263}{30240} & \frac{-863}{725760} & \cdots & 0 & 1 \\
\frac{1139}{45360} & \frac{47}{378} & \frac{11}{15120} & \frac{83}{2835} & \frac{-269}{15120} & \frac{11}{1890} & \frac{-37}{45360} & \cdots & 0 & 1 \\
\frac{137}{5376} & \frac{27}{224} & \frac{387}{8960} & \frac{17}{210} & \frac{-243}{8960} & \frac{9}{1120} & \frac{-29}{26880} & \cdots & 0 & 1 \\
\frac{143}{5670} & \frac{116}{945} & \frac{32}{945} & \frac{376}{2835} & \frac{29}{1890} & \frac{4}{945} & \frac{-2}{2835} & \cdots & 0 & 1 \\
\frac{3715}{145152} & \frac{725}{6048} & \frac{2125}{48384} & \frac{125}{1134} & \frac{3875}{48384} & \frac{235}{6048} & \frac{-275}{145152} & \cdots & 0 & 1 \\
\frac{41}{1680} & \frac{9}{70} & \frac{9}{560} & \frac{17}{105} & \frac{9}{560} & \frac{9}{70} & \frac{41}{1680} & \cdots & 0 & 1 \\
\vdots & \vdots & \vdots & \vdots & \vdots & \vdots & \vdots & \ddots & \vdots & \vdots \\
\frac{19087}{725760} & \frac{2713}{30240} & \frac{-15487}{241920} & \frac{293}{5670} & \frac{-6737}{241920} & \frac{263}{30240} & \frac{-863}{725760} & \cdots & 0 & 1 \\
\frac{41}{1680} & \frac{9}{70} & \frac{9}{560} & \frac{17}{105} & \frac{9}{560} & \frac{9}{70} & \frac{41}{1680} & \cdots & 1 & 1
\end{array}\right]\left[\begin{array}{c}
h f_{n} \\
h f_{n+\frac{1}{12}} \\
h f_{n+\frac{1}{6}} \\
h f_{n+\frac{1}{4}} \\
h f_{n+\frac{1}{3}} \\
h f_{n+\frac{5}{12}} \\
h f_{n+\frac{1}{2}} \\
\vdots \\
h f_{n+\frac{1}{12}} \\
h f_{n+\frac{1}{2}}
\end{array}\right]}
\end{aligned}
$$

\section{Real Life Problems}

\subsection{Problem 1: (SIR MODEL)}

The SIR model is an epidemiological model that computes the theoretical numbers of people infected with a contagious illness in a closed population over time. The name of this class of models derives from the fact that they involves coupled equations relating the number of susceptible people $S(t)$, number of people infected $I(t)$ and the number of people who have recovered $\mathrm{R}(\mathrm{t})$. This is a good and simple model for many infectious diseases including measles, mumps and rubella [13-15]. The SIR model is described by the three coupled equations.

$$
\begin{aligned}
& \frac{\mathrm{d} s}{\mathrm{~d} t}=\mu(1-S)-\beta I S \\
& \frac{\mathrm{d} I}{\mathrm{~d} t}=-\mu I-\gamma I+\beta I S \\
& \frac{\mathrm{d} s}{\mathrm{~d} t}=-\mu R+\gamma I
\end{aligned}
$$

where $\mu, \gamma$ and $\beta$ are positive parameters. Define $y$ to be

$$
y=S+I+R
$$

Adding Equations (11)-(13), we obtain the following evolution equations for $y$

$$
y^{\prime}=\mu(1-y)
$$

Taking $\mu=0.5$ and attaching an initial condition $y(0)=0.5$ (for a particular closed population), we obtain,

$$
y^{\prime}(t)=0.5(1-y), y(0)=0.5
$$

whose exact solution is,

$$
y(t)=1-0.5 \mathrm{e}^{-0.5 t}
$$

Applying our new half step numerical scheme (8) to solve SIR model simplified as (17) gives results as shown in Table 1.

\subsection{Problem 2 (Growth Model)}

Let us consider the differential equation of the form;

$$
\frac{\mathrm{d} N}{\mathrm{~d} t}=\alpha N, N(0)=1000, t \in[0,1]
$$

Equation (18) represents the rate of growth of bacteria in a colony. We shall assume that the model grows continuously without restriction. One may ask; how many bacteria are in the colony after some minutes if an individual produces an offspring at an average growth rate of 0.2 ? We also assume that $N(t)$ is the population size at time $t$ (Table 2).

The theoretical solution of (18) is given by;

$\mathrm{s}$

Note that, growth rate $\alpha=0.2$ in (18). 
Table 1. Showing results for SIR model problem.

\begin{tabular}{ccccc}
\hline $\mathrm{X}$ & Exact Result & Computed Solution & Error in half step method & Error in Sunday et al. \\
\hline 0.1 & 0.5243852877496430 & 0.5243852877496429 & $1.110223 \mathrm{e}-016$ & $5.574430 \mathrm{e}-012$ \\
0.2 & 0.5475812909820202 & 0.5475812909820202 & $0.000000 \mathrm{e}+000$ & $3.946177 \mathrm{e}-012$ \\
0.3 & 0.5696460117874711 & 0.5696460117874710 & $1.110223 \mathrm{e}-016$ & $8.183232 \mathrm{e}-012$ \\
0.4 & 0.5906346234610092 & 0.5906346234610089 & $2.220446 \mathrm{e}-016$ & $3.436118 \mathrm{e}-011$ \\
0.5 & 0.6105996084642976 & 0.6105996084642973 & $3.330669 \mathrm{e}-016$ & $1.929743 \mathrm{e}-010$ \\
0.6 & 0.6295908896591411 & 0.6295908896591410 & $1.110223 \mathrm{e}-016$ & $1.879040 \mathrm{e}-010$ \\
0.7 & 0.6476559551406433 & 0.6476559551406429 & $4.440892 \mathrm{e}-016$ & $1.776835 \mathrm{e}-010$ \\
0.8 & 0.6648399769821805 & 0.6648399769821799 & $5.551115 \mathrm{e}-016$ & $1.724676 \mathrm{e}-010$ \\
0.9 & 0.6811859241891134 & 0.6811859241891132 & $2.220446 \mathrm{e}-016$ & $1.847545 \mathrm{e}-010$ \\
1.0 & 0.6967346701436834 & 0.6967346701436828 & $5.551115 \mathrm{e}-016$ & $3.005770 \mathrm{e}-010$ \\
\hline
\end{tabular}

Table 2. Showing results for growth model problem.

\begin{tabular}{ccccc}
\hline $\mathrm{X}$ & Exact Result & Computed Solution & Error in half step method & Error in Sunday et al. \\
\hline 0.1 & 1020.2013400267558 & 1020.201340026755 & $0.000000 \mathrm{e}+000$ & $1.830358 \mathrm{e}-011$ \\
0.2 & 1040.8107741923882 & 1040.8107741923882 & $0.000000 \mathrm{e}+000$ & $1.250555 \mathrm{e}-011$ \\
0.3 & 1061.8365465453596 & 1061.8365465453596 & $0.000000 \mathrm{e}+000$ & $1.227818 \mathrm{e}-011$ \\
0.4 & 1083.2870676749587 & 1083.2870676749585 & $2.273737 \mathrm{e}-013$ & $3.137757 \mathrm{e}-011$ \\
0.5 & 1105.1709180756477 & 1105.1709180756475 & $2.273737 \mathrm{e}-013$ & $2.216893 \mathrm{e}-010$ \\
0.6 & 1105.1709180756477 & 1127.4968515793755 & $2.273737 \mathrm{e}-013$ & $2.060005 \mathrm{e}-010$ \\
0.7 & 1150.2737988572273 & 1150.2737988572271 & $2.273737 \mathrm{e}-013$ & $2.171419 \mathrm{e}-010$ \\
0.8 & 1173.5108709918102 & 1173.5108709918102 & $0.000000 \mathrm{e}+000$ & $2.216893 \mathrm{e}-010$ \\
0.9 & 1197.2173631218102 & 1197.2173631218102 & $0.000000 \mathrm{e}+000$ & $2.744400 \mathrm{e}-010$ \\
1.0 & 1221.4027581601699 & 1221.4027581601699 & $0.000000 \mathrm{e}+000$ & $4.899903 \mathrm{e}-010$ \\
\hline
\end{tabular}

Applying our new half step numerical scheme (8) to solve the Growth model (17) gives results as shown in Table 2 [16].

\subsection{Problem 3 (Decay Model)}

A certain radioactive substance is known to decay at the rate proportional to the amount present. A block of this substance having a mass of $100 \mathrm{~g}$ originally is observed. After 40 mins, its mass reduced to $90 \mathrm{~g}$. Find an expression for the mass of the substance at any time and test for the consistency of the block integrator on this problem for $t \in[0,1]$.

The problem has a differential equation of the form;

$$
\frac{\mathrm{d} N}{\mathrm{~d} t}=-\alpha N, N(0)=100, t \in[0,1]
$$

where $N$ represents the mass of the substance at any time $t$ and $\alpha$ is a constant which specifies the rate at which this particular substance decays. Note that,

$$
f(0)=100 \mathrm{~g}, t=40 \mathrm{mins}, f(40)=90 \mathrm{~g}
$$

Since for any growth/decay problem,

$$
\begin{gathered}
f(t)=f(0) \mathrm{e}^{\alpha t} \\
90=100 \mathrm{e}^{40 \alpha} \\
\alpha=\frac{(\ln 9-\ln 10)}{40}=-0.0026
\end{gathered}
$$

Thus, the theoretical solution to (20) is given by,

$$
f(t)=100 \mathrm{e}^{-0.0026 t}
$$

which is also the expression for the mass of the substance at any time $t$.

Applying our new half step numerical scheme (8) to solve the Growth model (22) gives results as shown in Table $3[16,17]$ (Table 3).

\section{Discussion of the Result}

We have considered three real-life model problems to test the efficiency of our method. Problems 1 and 2 and 3 were solved by Sunday et al. [17]. They proposed an order six block integrator for the solution of first-order ordinary differential equations. Our half-step block method gave better approximation as shown in Tables 
Table 3. Showing results for decay model problem.

\begin{tabular}{ccccc}
\hline $\mathrm{X}$ & Exact Result & Computed Solution & Error in half step method & Error in Sunday et al. \\
\hline 0.1 & 99.9740033797070850 & 99.9740033797070850 & $0.000000 \mathrm{e}+000$ & $0.000000 \mathrm{e}+000$ \\
0.2 & 99.9480135176568470 & 99.9480135176568330 & $1.421085 \mathrm{e}-014$ & $1.421085 \mathrm{e}-014$ \\
0.3 & 99.9220304120923400 & 99.9220304120923400 & $0.000000 \mathrm{e}+000$ & $0.000000 \mathrm{e}+000$ \\
0.4 & 99.8960540612571460 & 99.8960540612571460 & $0.000000 \mathrm{e}+000$ & $0.000000 \mathrm{e}+000$ \\
0.5 & 99.8700844633952300 & 99.8700844633952440 & $1.421085 \mathrm{e}-014$ & $0.000000 \mathrm{e}+000$ \\
0.6 & 99.8441216167510670 & 99.8441216167510820 & $1.421085 \mathrm{e}-014$ & $0.000000 \mathrm{e}+000$ \\
0.7 & 99.8181655195695610 & 99.8181655195695750 & $1.421085 \mathrm{e}-014$ & $0.000000 \mathrm{e}+000$ \\
0.8 & 99.7922161700960970 & 99.7922161700960970 & $0.000000 \mathrm{e}+000$ & $0.000000 \mathrm{e}+000$ \\
0.9 & 99.7662735665764730 & 99.7662735665764730 & $0.000000 \mathrm{e}+000$ & $0.000000 \mathrm{e}+000$ \\
1.0 & 99.7403377072569700 & 99.7403377072569700 & $0.000000 \mathrm{e}+000$ & $0.000000 \mathrm{e}+000$ \\
\hline
\end{tabular}

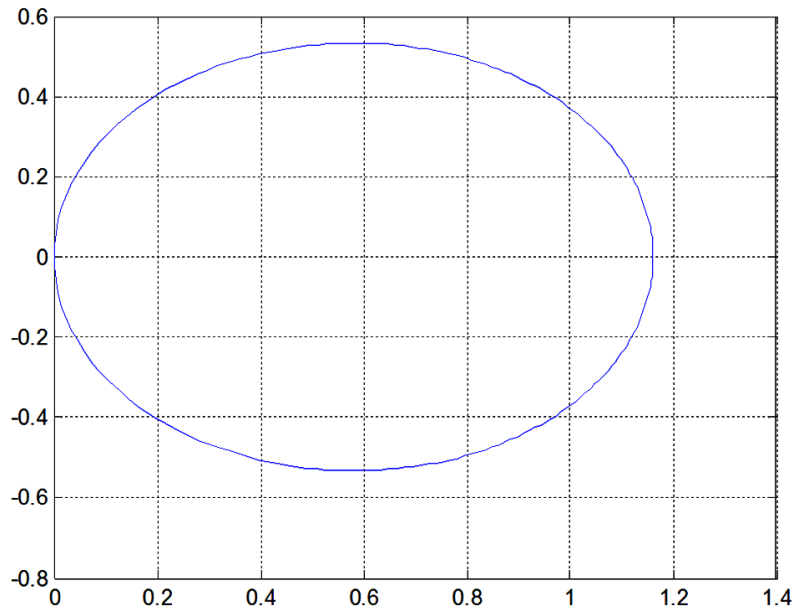

Figure 1. Showing region of absolute stability of our method.

1-3 because the iteration per step in the new method was lower than the method proposed by [17]. Our method was found to be zero stable, consistent and converges. Figure 1 shows the region of absolute stability. From the numerical examples, we could safely conclude that our method gave better accuracy than the existing methods.

\section{REFERENCES}

[1] A. O. Adesanya, M. R. Odekunle, and A. A. James, "Order Seven Continuous Hybrid Methods for the Solution of First Order Ordinary Differential Equations," Canadian Journal on Science and Engineering Mathematics, Vol. 3 No. 4, 2012, pp. 154-158.

[2] A. M. Badmus and D. W. Mishehia, "Some Uniform Order Block Methods for the Solution of First Ordinary Differential Equation," J. N.A.M. P, Vol. 19, 2011, pp 149154.

[3] J. Fatokun, P. Onumanyi and U. W. Sirisena, "Solution of First Order System of Ordering Differential Equation by Finite Difference Methods with Arbitrary," J.N.A.M.P, 2011, pp. 30-40.
[4] B. I. Zarina and S. I. Kharil, "Block Method for Generalized Multistep Method Adams and Backward Differential Formulae in Solving First Order ODEs," MATHEMATIKA, 2005, pp. 25-33.

[5] G. Dahlquist, "A Special Stability Problem for Linear Multistep Methods," BIT, Vol. 3, 1963, pp. 27-43.

[6] P. Onumanyi, U. W. Sirisena and S. A. Jator, "Solving Difference Equation," International Journal of Computing Mathematics, Vol. 72, 1999, pp. 15-27.

[7] E. A. Areo, R. A. Ademiluyi and P. O. Babatola, "ThreeStep Hybrid Linear Multistep Method for the Solution of First Order Initial Value Problems in Ordinary Differential Equations,” J.N.A.M.P, Vol. 19, 2011, pp. 261-266.

[8] E. A. Ibijola, Y. Skwame and G. Kumleng, "Formation of Hybrid of Higher Step-Size, through the Continuous Multistep Collocation," American Journal of Scientific and Industrial Research, Vol. 2, 2011, pp. 161-173.

[9] H. S. Abbas, "Derivation of a New Block Method Similar to the Block Trapezoidal Rule for the Numerical Solution of First Order IVPs," Science Echoes, Vol. 2, 2006, pp. $10-24$.

[10] Y. A. Yahaya and G. M. Kimleng, "Continuous of TwoStep Method with Large Region of Absolute Stability," J.N.A.M.P, 2007, pp. 261-268.

[11] A. O. Adesanya, M. R. Odekunle and A. A. James, "Starting Hybrid Stomer-Cowell More Accurately by Hybrid Adams Method for the Solution of First Order Ordinary Differential Equation," European Journal of Scientific Research, Vol. 77 No. 4, 2012, pp. 580-588.

[12] S. Abbas, "Derivations of New Block Method for the Numerical Solution of First Order IVPs," International Journal of Computer Mathematics, Vol. 64, 1997, p. 325.

[13] W. B. Gragg and H. J. Stetter, "Generalized Multistep Predictor-Corrector Methods," Journal of Association of Computing Machines, Vol. 11, No. 2, 1964, pp. 188-209. http://dx.doi.org/10.1145/321217.321223

[14] J. B. Rosser, "A Runge-Kutta Method for All Seasons," SIAM Review, Vol. 9, No. 3, 1967, pp. 417-452. http://dx.doi.org/10.1137/1009069

[15] R. E. Mickens, "Nonstandard Finite Difference Models of Differential Equations," World Scientific, Singapore, 1994. 
[16] O. D. Ogunwale, O. Y. Halid and J. Sunday, "On an Alternative Method of Estimation of Polynomial Regression Models," Australian Journal of Basic and Applied Sciences, Vol. 4, No. 8, 2010, pp. 3585-3590.
[17] J. Sunday, M. R. Odekunleans and A. O. Adeyanya, "Or der Six Block Integrator for the Solution of First-Order Ordinary Differential Equations," IJMS, Vol. 3, No. 12013 , pp. 87-96. 